\title{
A Cost-Effective Methodology for a Run-by-Run EWMA Controller
}

Ruey-Shan Guo and Li-Shia Huang

Department of Industrial Management and Business Administration National Taiwan University
Argon Chen

Graduate Institute of Industrial Engineering

National Taiwan University
Jin-Jung Chen

Department of Mechanical Engineering

National Taiwan University
Abstract - In this paper, we present a cost-effective methodology for a run-by-run EWMA controller. This controller is an integrated approach that combines the advantages of statistical process control and feedback control. It adjusts the equipment settings only when the control chart detects an abnormal trend. Using simulations, we take into considerations the control sensitivity, robustness, and adjustment number required to determine an optimal weight for a minimum cost. As the simulation results demonstrate, the cost-effective run-by-run controller is able to keep a drifting process outputs close to the target with only few runs of adjustment.

\section{INTRODUCTION}

The Run by Run (RbR) process controller, originally developed by an MIT research group [1][2], combines statistical process control (SPC) and feedback control. Here, SPC is used to decide the control of the next run to be "gradual" or "rapid." In the gradual mode of control, the constant terms of linear models, characterizing the underlying process, are updated based on exponentially weighted moving average (EWMA) algorithm. The gradual mode of the RbR controller is, in essence, a continuous EWMA controller.

The EWMA controller is an effective controller for controlling processes when there are process drifts and small shifts and when the adjustment of equipment settings is reasonably easy and inexpensive [3]. However, when the cost of adjusting the equipment settings is significant or the underlying abnormal patterns are not certain, a more rigorous detection rule should be exercised as to when to control and when not to control. Our goal in this paper is then to present a "detection and control" methodology for the EWMA controller so that it can be more cost-effective.

Figure 1 illustrates the proposed detection and control system architecture. As can be seen, there are two main modules in this system: SPC module and feedback control module. In the SPC module, the goal is to distinguish between common causes of deviations and special causes of deviations. Only when there is an evidence indicating that the abnormal deviation is statistically significant, will the control actions be taken. In the feedback control module, the goal is to compensate for the deviation by adjusting the equipment settings of the next run. This detection and control process is then repeated on a RbR basis. Detailed algorithm about each module and the simulation results are described in the following sections.

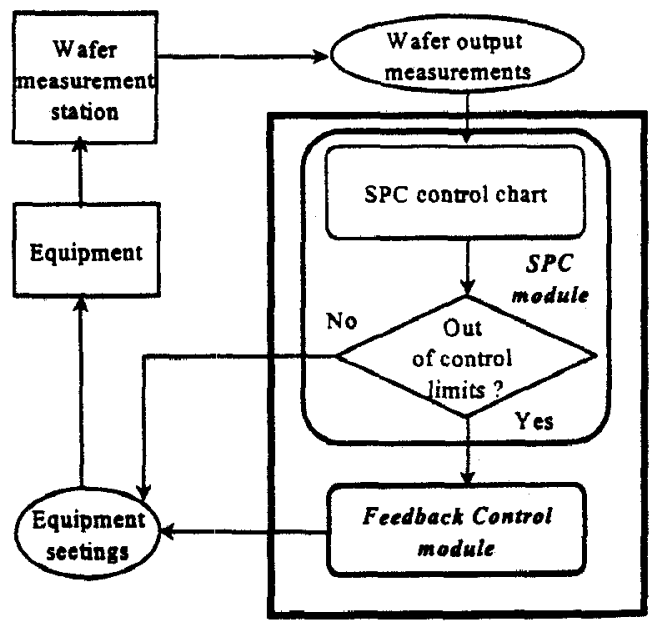

Figure I Detection and control system architecture.

\section{STATISTICAL PROCESS CONTROL}

To enhance the detection sensitivity, the characteristics of different types of control charts are studied. In general, there are four types of control charts and their difference can be characterized by the weighting scheme on the historical data (Figure 2).

- Shewhart control chart: Each control point uses only the current measurements so a $100 \%$ weight is assigned to the current data.

- CUSUM control chart: Each control point uses all the historical data by assigning equal weights to all 
historical data.

- Moving average control chart: Each control point uses the latest $m$ ( $m$ is a constant) data points by assigning equal weights to the latest $m$ points.

- EWMA control chart: Each control point uses all the historical data but the more recent the data, the higher the weight. As shown in (1), the weight is exponentially decreased as the data point ages.

$$
E_{t}=W e_{t}+(1-W) E_{t-1}=\sum_{j=1}^{t} W(1-W)^{t-j} e_{j}
$$
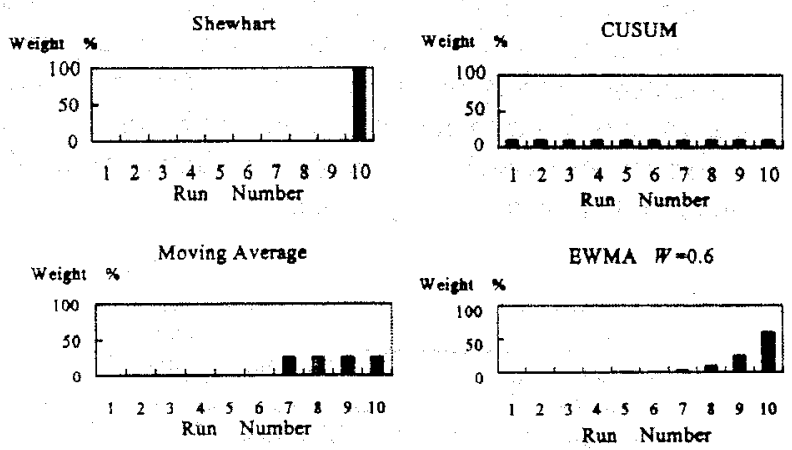

Figure 2 Weighting schemes of different control charts.

In the traditional SPC, the process is assumed to be static and the process inputs are usually kept unchanged. In our detection and control scheme, inputs (equipment settings) might change from run to nu, and the direct plotting of output values makes no sense. The correct way is to plot the residuals between the predicted output values and measured output values. This is shown in (2) and (3).

$$
\hat{Y}_{t}=a_{k-1}+b X_{t}
$$

$\hat{Y}_{t} \quad$ : predicted output value ( $t$ is the current run no)

$a_{k-1}$ :model constant term ( $k$ is the model tuning no)

$b \quad:$ model slope term (assumed fixed)

$X_{t} \quad$ : equipment settings

$Y_{t} \quad$ : wafer output measurement

$$
e_{t}=Y_{t}-\hat{Y}_{t}
$$

Figure 3 illustrates the functional block diagram of our detection and control framework. In the SPC module, once the residual for the current run is calculated, a Shewhart control chart is first used to detect if there is a large deviation. If there is a statistical evidence indicating that the process has a large shift $\left(\left|e_{t}\right|>3 \sigma\right)$, then we might stop the process for further diagnosis. If there is no large shift, an EWMA control chart is then used to detect if there is a small deviation. If there is a statistical evidence showing that the process has a small deviation $\left(\left|E_{t}\right|>3 \sigma \sqrt{W /(2-W)}\right)$, then control actions will be taken. Otherwise, the current process is deemed stable and no control action is needed.

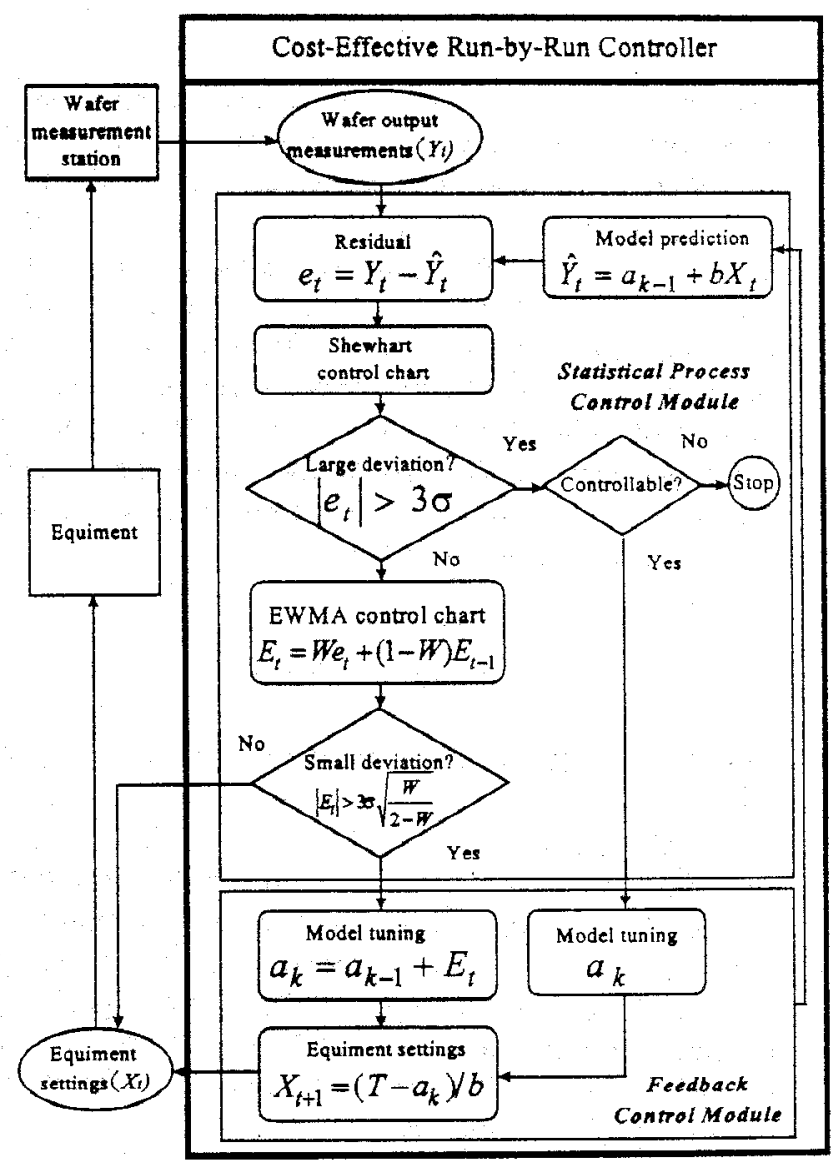

Figure 3 Functional block diagram of a detection and control framework.

To demonstrate the detection sensitivity, simulations are performed. As shown in Figures 4 and 5, a process has a large shift in run 16 and has a small drift from run 11 . The Shewhart control chart is able to detect the shift but not the drift. This is due to the fact that the Shewhart control chart uses only the last measurement data. On the other hand, the EWMA control chart with a weighting factor $W=$ 0.2 is able to detect the drift but not the shift. This result indicates that the EWMA control chart is more sensitive to small deviations; however, the weighting factor must be carefully chosen. 


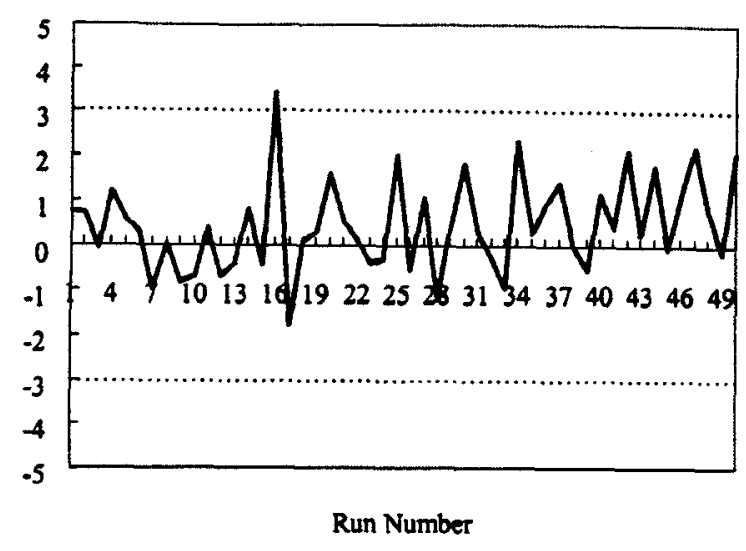

Figure 4 Shewhart control chart.

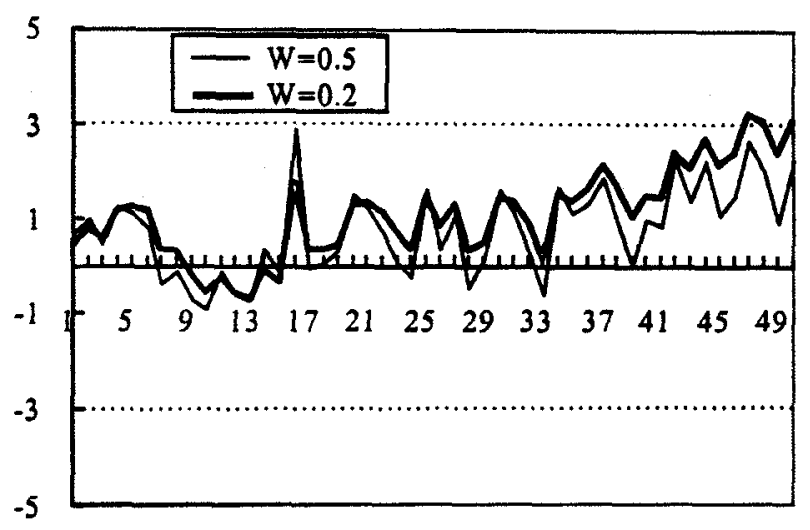

Run Number

Figure 5 EWMA control chart.

One way to decide the optimal weighting factor for the EWMA control chart is to balance between the sensitivity and robustness. By deriving the run length distribution using Markov-chain formulation, we are able to characterize the sensitivity and robustness under different weighting factors and an optimal weighting factor can be chosen accordingly [4]. However, in our detection and control scheme, the EWMA control scheme serves not only as a detection device but also a deviation compensator. An optimal weighting factor must be chosen based on the overall control efficiency. In the next section, a costeffective methodology is proposed.

\section{FEEDBACK CONTROL}

In the feedback control module, we use the EWMA values $\left(E_{t}\right)$ to fine tune the constant terms of the control models. Then the equipment settings of the next run can be obtained by using the updated control models. Equations (4) and (5) show the calculations.

$$
\begin{aligned}
& a_{k}=a_{k-1}+E_{t} \\
& X_{t+1}=\frac{\left(T-a_{k}\right)}{b} \quad \text { ( } T \text { is target value) }
\end{aligned}
$$

As mentioned earlier, to ensure the detection and control to be cost-effective, the weighting factor ( $W$ in the EWMA formulation must be chosen carefully. If we can assume the underlying small deviations are due to process drifts, then it is possible to characterize the detection and control performance against different values of weighting factor and drift speed.

Our approach is to use Monte Carlo simulations to evaluate the required adjustment number, the control sensitivity, and the robustness against different values of weighting factor and drift speed. Here, the control sensitivity is characterized by MSE (mean square error) divided by natural variance; and the robustness is evaluated by $M S E O$ (mean square error when there is no drift) divided by natural variance. Since both $M S E$ and $M S E O$, as shown in (6), is proportional to the quality loss as defined by Taguchi, their values should be as small as possible. Figure 6 illustrates the result of one case study in which the drift speed is 0.02 (i.e., $2 \sigma$ after 100 runs) and 1000 runs are simulated.

$$
M S E=\frac{1}{n} \sum_{i=1}^{n}\left(Y_{i}-T\right)^{2}
$$

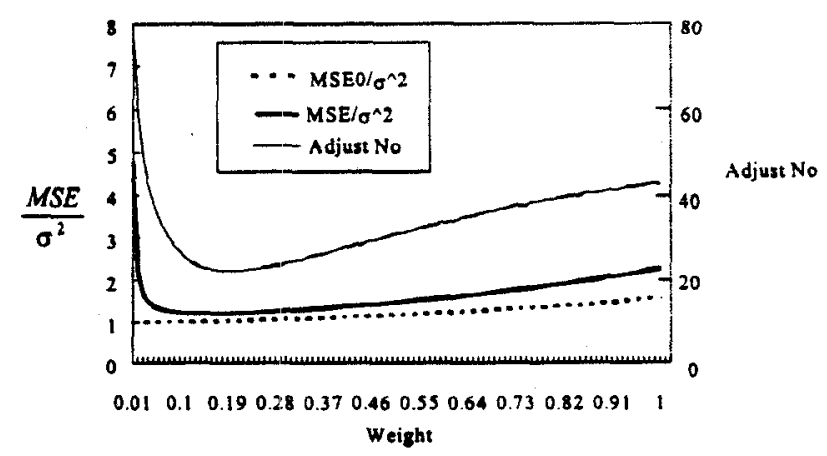

Figure 6 Correlation between weighting factor, MSE, $M S E 0$, and adjustment number.

Finally, a total cost criterion is proposed to select the optimal weighting factor for the cost-effective EWMA controller. If the cost coefficients for MSE, MSEO and adjustment number are given, as shown in (7), the total cost can be obtained, and the optimal weighting factor is the one 
that minimizes the total cost.

$$
\text { Cost }=\mathrm{C}_{1}\left(M S E / \sigma^{2}\right)+\mathrm{C}_{2}\left(M S E 0 / \sigma^{2}\right)+\mathrm{C}_{3} \text { (Adjustment no) }
$$

In order to demonstrate the effectiveness of the proposed detection and control methodology, a simulation is performed and results are shown in Figures 7 and 8 . In Figure 7, we see that a drifting process is able to be brought back to the target value using the cost-effective EWMA controller. In Figure 8, we see that there are only four outof-control signals (and thus four feedback control adjustments) in 200 rums.

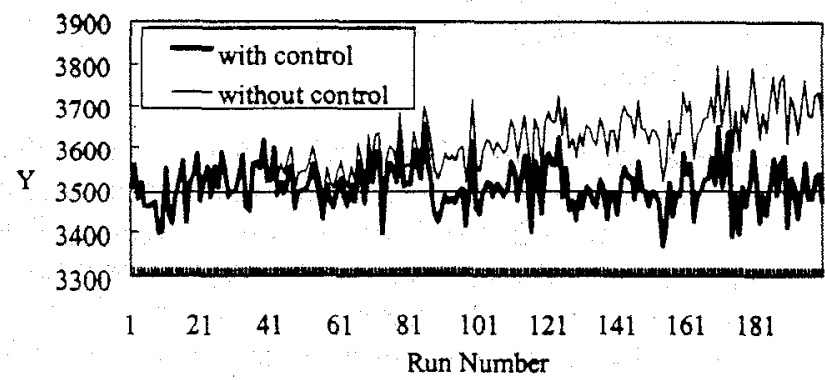

Figure 7 Simulated drift process with and without control.

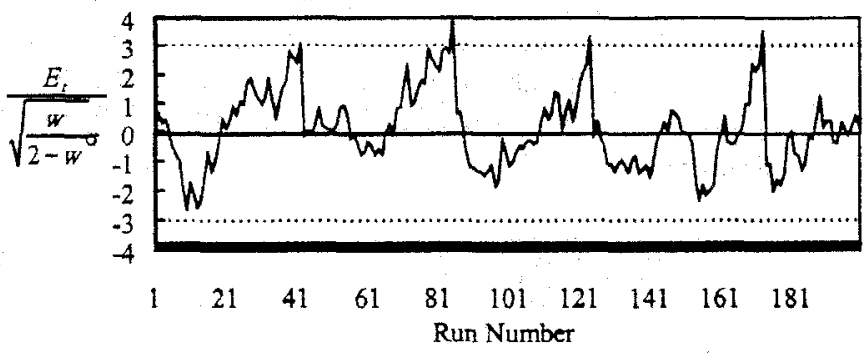

Figure 8 EWMA chart for the simulated drifting process with cost-effective EWMA control.

To further demonstrate the controller's characteristics, more simulations are performed and results are shown in Table 1. Here, for a given drift speed, the optimal weighting factor is chosen for each controller (cost-effective and continuous EWMA controllers) and 1000 runs are simulated. As can be seen, under different drift conditions, the cost-effective EWMA controller is able to, with much less frequent adjustment, reduce the $M S E$ and the result is comparable to that of using a continuous EWMA controller. Based on the results in Table 1, we can also conclude that a continuous EWMA controller, in general, is suitable for situations where the drift speed is high and the adjustment of equipment settings is relatively easy and inexpensive. However, if the drift speed is small or uncertain while adjustment cost is significant, then a detection and control scheme will be more cost-effective than a continuous control scheme.

\section{CONCLUSIONS}

In this paper, we present a detection and control scheme for the RbR EWMA controller. The controller combines the advantages of SPC and feedback control. In specific, its optimal weighting factor is chosen based on the overall control efficiency by considering the control sensitivity, the robustness, and the adjustment number. As the simulation results demonstrate, the cost-effective EWMA controller is able to keep a drifting process outputs close to the target with only few runs of adjustment. Future plans include the application to fab process control and a control scheme for large process deviations.

\section{REFERENCES}

[1] E. Sachs, R. Guo, S. Ha, A. Hu, "Process Control System for VLSI Fabrication," IEEE Trans. Semi. Manufacturing, vol. 4, May 1991.

[2] E. Sachs, A. Hu, A. Ingolfsson, "Run by Run Process Control: Combining SPC and Feedback Control," IEEE Trans. Semi. Marufacturing, vol. 8, Feb. 1995.

[3] T. Smith, D. Boning, "A Self-Tuning EWMA Controller Utilizing Artificial Neural Network Function Approximation Techniques," IEMT, Austin, Oct. 1996.

[4] L. Huang, "A Cost-Effective Methodology for a Runby-Run EWMA Controller," MBA thesis, Department of Industrial Management and Business Administration, National Taiwan University, June 1997

\begin{tabular}{|c|c|c|c|c|c|c|c|c|}
\hline & \multicolumn{3}{|c|}{ Cost-Effective EWMA Controller } & \multicolumn{4}{c|}{ Continuous EWMA Controller } \\
\hline Drift speed & $W$ & $M S E / \sigma^{2}$ & Adjust. no & $M S E 0 / \sigma^{2}$ & $W$ & $M S E / \sigma^{2}$ & Adjust. no & $M S E 0 / \sigma^{2}$ \\
\hline 0.001 & 0.02 & 1.0225 & 3 & 1.0022 & 0.02 & 1.0126 & 999 & 1.0050 \\
\hline 0.01 & 0.09 & 1.1251 & 15 & 1.0145 & 0.07 & 1.0566 & 999 & 1.0363 \\
\hline 0.02 & 0.15 & 1.2085 & 23 & 1.0231 & 0.11 & 1.0912 & 999 & 1.0582 \\
\hline 0.05 & 0.27 & 1.4178 & 40 & 1.0667 & 0.2 & 1.1735 & 999 & 1.1111 \\
\hline
\end{tabular}

Table 1 Performance comparison between the cost-effective EWMA controller and continuous EWMA controller. 